\title{
Malignant peritoneal mesothelioma: clinical aspects, and therapeutic perspectives
}

\section{Stergios Boussios ${ }^{a}$, Michele Moschetta ${ }^{b}$, Afroditi Karathanasia, Alexandros K. Tsiourisc, Foivos S. Kanellos ${ }^{\text {, }}$ Konstantina Tatsi ${ }^{d}$, Konstantinos H. Katsanos ${ }^{\mathrm{e}}$, Dimitrios K. Christodoulou ${ }^{\mathrm{e}}$}

Medway NHS Foundation Trust, Kent, UK; Sarah Cannon Research Institute, London, UK; University of Ioannina, Greece; General Hospital G. Hatzikosta, Ioannina, Greece

\section{Abstract}

${ }^{\mathrm{a}}$ Medway NHS Foundation Trust, Kent, UK (Stergios Boussios, Afroditi Karathanasi); 'brug Development Unit, Sarah Cannon Research Institute, London, UK (Michele Moschetta); 'Department of Biological Applications \& Technology, University of Ioannina, Ioannina, Greece (Alexandros K. Tsiouris, Foivos S. Kanellos); ${ }^{\mathrm{d} G y n e c o l o g y}$ Unit, General Hospital “G. Hatzikosta”, Ioannina, Greece (Konstantina Tatsi); ${ }^{\mathrm{e} D}$ Department of Gastroenterology, University Hospital of Ioannina, Faculty of Medicine, School of Health Sciences, University of Ioannina, Greece (Konstantinos H. Katsanos, Dimitrios K. Christodoulou)

Conflict of Interest: None

Correspondence to: Dr Stergios Boussios MD, PhD, Consultant Medical Oncologist, Medway NHS Foundation Trust, Windmill Road, Gillingham, Kent, ME7 5NY, UK; e-mail: stergiosboussios@gmail.com, and stergios.boussios@nhs.net

Received 30 May 2018; accepted 18 July 2018; published online 14 September 2018

DOI: https://doi.org/10.20524/aog.2018.0305

\section{Introduction}

Mesothelioma arises primarily from the mesothelial cells of the serosal membrane lining the pleural, peritoneal and pericardial cavities, and the tunica vaginalis. Among the various types of mesothelioma, diffuse malignant peritoneal mesothelioma (MPM) accounts for nearly one-fourth of cases, with an estimated annual incidence of 2500 cases worldwide [1]. MPM is a heterogeneous, aggressive tumor mainly caused by exposure to asbestos or other carcinogens such as talcum; $33 \%$ of diagnosed patients have a history of asbestos exposure, a clearly lower rate than that in patients with malignant pleural mesothelioma [2]. Chronic peritonitis is a less clear risk factor [3]. Patients with MPM are more commonly female, of younger age, and have a better prognosis than those with pleural mesothelioma [4]. The disease typically presents with non-specific features, including abdominal pain, 
palpable pelvic masses, altered bowel habit, tumor nodules of variable size located diffusely throughout the peritoneal cavity, and massive malignant ascites [5]. The morphologic appearance ranges from epithelioid to sarcomatoid and biphasic subtypes. Among these, epithelioid tumors have a better prognosis [6].

Cytoreductive surgery (CRS) with hyperthermic intraperitoneal chemotherapy (HIPEC) is a treatment option for patients with good performance status and potentially resectable disease $[7,8]$. Despite improved outcomes and the comparatively long-term survival, the combined therapy is associated with significant perioperative morbidity in more than half of patients [9]. Treatment for inoperable MPM consists of palliative chemotherapy with pemetrexed, cisplatin and gemcitabine, either alone or in combination, whereas second-line therapy is currently not defined [10].

Given the limited therapeutic options in the advanced setting, the prognosis remains dismal, with median survival reported to be one year in a historical series [11] and 13 months in a multicenter registry study [12]. This article provides an overview of our current knowledge concerning the epidemiology, clinical presentation, diagnosis, pathology, genetics, therapeutic interventions and prognostic factors of MPM.

\section{Materials and methods}

The PubMed database was searched using the terms "peritoneal, mesothelioma", "pleural, mesothelioma", "immunohistochemistry, mesothelioma", "genomic profiling, mesothelioma", "treatment, mesothelioma", and "prognosis, mesothelioma". Publications between September 1999 and February 2018 were eligible for inclusion. Case series of patients with MPM describing diagnostic and therapeutic considerations along with targeted approaches were also included in this study.

\section{Epidemiology}

The incidence of MPM has been increasing since the 1970s and its mortality increased by $2.78 \%$ each year from 1994-2008 [3,13]. It currently represents the second most common site of malignant mesothelioma, accounting for $20 \%$ of reported cases [14]. Slight differences in incidence rates among western countries have been described, ranging from 0.5-3 cases per million [15]. The UK reports the highest incidence, estimated at 3.6 and 0.7 cases per 100,000 people for men and women, respectively [16].

The reported female-to-male ratio for MPM is 0.70 [17]. Interestingly, female patients live significantly longer than male patients, which suggests that sex has substantial prognostic relevance [18,19]. Patients with MPM are significantly younger, and have a shorter median overall survival (OS) than those with the pleural variant (mean age, 63.3 versus 70.8 years, respectively). In addition, females diagnosed with MPM live significantly longer than male patients; no racial predilection has been described [18].

\section{Etiology}

The main carcinogen leading to the development of MPM is asbestos; nevertheless, only $33-50 \%$ of patients diagnosed with MPM report prior exposure to asbestos [15]. The long latency between asbestos contact and the onset of mesothelioma implies that the incidence of this disease will continue to increase in the future [20]. Several additional environmental factors have been implicated, including talc, mica, erionite (volcanic ash), and thorotrast. In addition, Hodgkin's disease, chronic peritonitis and exposure to therapeutic radiation have been correlated with MPM [15].

\section{Clinical presentation}

MPM is usually diagnosed at an advanced stage, probably because disease manifestations are non-specific in the early stage. Clinical presentation is quite variable, depending on the extent of tumor spread within the abdominal cavity. Accumulation of ascites and growth of tumor masses result in abdominal distension in $30-80 \%$ of patients. Abdominal pain is the second most common presenting symptom in approximately $27-58 \%$ of cases [16]. The typical growth pattern of MPM is diffuse, rather than infiltrative [21]. Swelling of lymph nodes due to local involvement may lead to the obstruction of the superior vena cava, while an acute abdomen can be caused by malignant bowel obstruction or perforation[22]. Patients may also suffer from unspecific symptoms, such as early satiety, anorexia, weight loss, vomiting, constipation, and/or diarrhea. Less common complaints include new-onset hernia, fever of unknown origin and night sweats [23]. Nevertheless, approximately $8 \%$ of patients are diagnosed incidentally [24].

\section{Diagnostic considerations}

The precise diagnosis of MPM is based on a detailed medical history, followed by radiological, laboratory, and pathological examinations. Computed tomography (CT), magnetic resonance imaging (MRI), and positron-emission tomography (PET) are the main imaging modalities used; nevertheless, there is no pathognomonic imaging finding for MPM. CT scan may reveal a solid, heterogeneous, soft-tissue mass with irregular margins and demonstrate high contrast between the enhanced tumor and the non-enhanced ascites[25]. Peritoneal and mesenterial thickening are also frequent findings [12]. Since extra-abdominal spread is uncommon, further imaging is rarely indicated. Diffusion-weighted and dynamic gadoliniumenhanced MRI may demonstrate more accurately the extent of the disease, but these modalities are not implemented routinely[26]. PET scanning provides information about the stage of MPM for preoperative patients and allows the detection of lymph node involvement, which may be undetectable on CT scan [3]. PET can also detect potential recurrent disease with more sensitivity [27]. Based on cross-sectional imaging, 
the differential diagnosis for MPM may include peritoneal carcinomatosis, serous peritoneal, and ovarian carcinoma, as well as lymphomatosis and tuberculous peritonitis.

Biopsy is essential for establishing diagnosis and can be performed either radiographically or surgically. Laparoscopy represents a preferable diagnostic approach, considering its lower invasiveness and clear intraoperative assessment[12,28]. Whereas the wall of the mobile small bowel is typically not involved in peritoneal carcinomatosis, during either laparoscopy or laparotomy in patients with no medical history of abdominal operations, the serosal layer of the small bowel wall, consisting of mesothelial cells, is commonly diffusely involved. The mesentery is involved in both cases.

CA-125 and CA 15-3 baseline levels are elevated in 53.3\% and $48.5 \%$ of patients, respectively [29]. Serum mesothelinrelated protein has a higher sensitivity of $60 \%$ [30], whereas serum high-mobility group box 1 (HMGB1), hyaluronic acid, and osteopontin could be useful markers for monitoring disease recurrence [29].

\section{Histology and immunohistochemistry}

Histologically, MPM is divided into epithelioid, sarcomatoid, and biphasic subtypes. Among these, epithelioid is the most common, representing $75-90 \%$ of reported cases, and is associated with the best prognosis, as mentioned above[6]. Approximately 25\% of MPMs are biphasic, while the sarcomatoid subtype is extremely rare. The epithelioid subtype is composed of cells that resemble normal mesothelial cells in a predominantly tubulopapillary or trabecular pattern with uncommon mitotic figures. The biphasic subtype consists of both epithelioid and sarcomatoid elements, with each component contributing at least $10 \%$ of the overall histology[31]. The sarcomatoid subtype is composed of tightly arranged spindle cells with sporadic malignant osteoid, chondroid or muscular features. The prognosis of biphasic and sarcomatoid subtypes is significantly worse, similar to that of the corresponding pleural mesothelioma variants $[3,32]$.

The diagnosis of MPM based entirely on histologic patterns may be challenging; thus, immunohistochemical panels are usually used and can provide the more sensitive and specific information needed for an accurate diagnosis (Fig. 1). In addition, there is increasing interest in using markers relatively tissue-specific transcription factors. Furthermore, the optimal immunohistochemical panel for distinguishing MPM from ovarian serous tumors remains to be clearly defined. There is a general consensus that EMA, calretinin, CK5/6, Wilms tumor 1 (WT-1), HBME-1, thrombomodulin, podoplanin, mesothelin, and D2-40 are immunoreactive in MPM. However, WT1[33,34], D2-40 [35], calretinin [32], and cytokeratin 5/6 [36] can also be positive in the majority of serous carcinomas. On the other hand, TTF1, CEA, Ber-Ep4, LeuM1, B72.3, MOC31 and cluster of differentiation (CD) 15 are commonly expressed in adenocarcinoma rather than mesothelioma [37]. Overall, it is recommended to use at least two mesothelioma markers and two carcinoma markers [3,38].

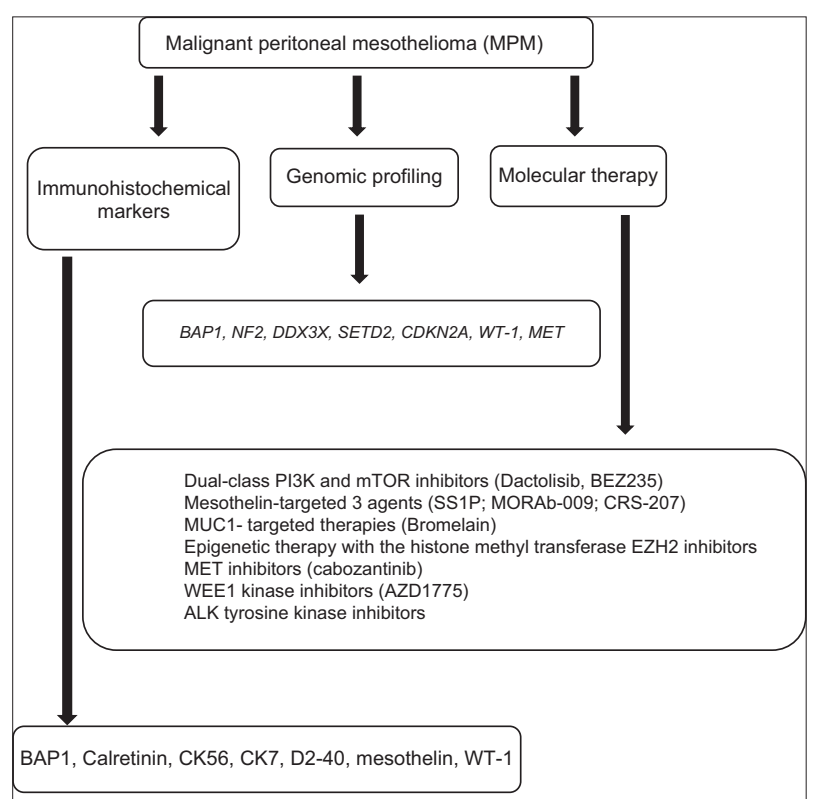

Figure 1 Immunohistochemical and molecular profiling and molecular therapies of malignant peritoneal mesothelioma (MPM)

\section{Genomic profiling of MPM}

The genetic alterations that drive mesotheliomas occur at distinct frequencies depending on the anatomic origin of the tumor. One study revealed chromosome 9p21 deletion more frequently in pleural than in peritoneal tumors ( $85 \%$ vs. $36 \%)$, whereas $5 \mathrm{p} 15$ and $7 \mathrm{p} 12$ gains were more common in peritoneal than in pleural tumors [39].

$B A P 1$ is a tumor-suppressor gene that enhances BRCA1mediated cell growth suppression, which plays a significant role in the organogenesis of the Müllerian system [40]. It is expressed in a variety of ovarian tumors, particularly serous carcinoma, and has been established as a sensitive and specific marker for ovarian serous carcinomas, as well as an important parameter in the differential diagnosis with MPM. Its sensitivity ranges between $90 \%$ and $100 \%$ [41]. The expression of $P A X 8$ in the Müllerian lesions is generally strong and diffuse. However, $P A X 8$ is expressed in only a small minority of MPMs, according to the limited relevant literature available [41].

$B A P 1$ is lost in almost $50 \%$ of pleural mesotheliomas and two thirds of MPMs, but in less than $1 \%$ of high-grade serous carcinomas [42]. Table 1 depicts the available studies that reported a loss of BAP1 in MPM. One study identified that frequent loss of BAP1 immunostaining in MPM did not affect significantly the outcome [43]. Therefore, loss of nuclear BAP1 confirm the diagnosis of MPM but does not offer prognostic information. On the other hand, preservation of BAP1 cannot distinguish MPM from other Müllerian lesions, because of its suboptimal sensitivity $[42,44]$. Cytogenetically, $40-70 \%$ of both pleural and peritoneal mesotheliomas harbor a loss of $9 \mathrm{p}$ including cyclin-dependent kinase activator inhibitor $2 \mathrm{~A}$ (CDKN2A), or 22q including NF2 [43]. The combination of homozygous CDKN2A deletion and hemizygous NF2 loss in MPM is an independent prognostic factor, associated with 
Table 1 BAP1 loss in peritoneal mesothelioma

\begin{tabular}{lccc}
\hline Author [ref.] & Publication year & N & BAP1 loss in peritoneal mesothelioma, (\%) \\
\hline Andrici et al [42] & 2016 & 9 & $6(67 \%)$ \\
Singhi et al [43] & 2016 & 86 & $49(57 \%)$ \\
Kato et al [116] & 2016 & 11 & $3(27 \%)$ \\
Shinozaki-Ushiku et al [44] & 2017 & 32 & $17(53 \%)$ \\
Tandon et al [117] & 2018 & 181 & $99(55 \%)$ \\
\hline BAP1, BRCAl-associated protein-1 & &
\end{tabular}

BAP1, BRCA1-associated protein-1

shorter progression-free survival (PFS) and OS. In addition, tumor suppressor gene BAP1 mutation can predispose to MPM. An array comparative genomic hybridization study involving 33 MPM patients revealed focal deletions affecting $B A P 1$ and $C D K N 2 A$ in $61 \%$ and $33 \%$ of cases, respectively, suggesting that similar genetic alterations drive MPM and pleural mesothelioma but at different frequencies [45]. The genomic profiling of patients with MPM is summarized in Fig. 1.

Limited evidence is currently available for the functional role of specific microRNAs (miRNAs) in MPM [46]. It has been suggested that miR-380-5p-a miRNA normally negligibly expressed in telomerase-positive MPM tissues-may interfere with telomerase activity and promotes cell growth impairment and induction of apoptosis in relevant models of MPM [47].

\section{Treatment options}

\section{Surgical therapy}

Given the rarity of MPM, data on the best treatment strategies have been based mostly on single institutional retrospective reports [15]. In the absence of coexisting medical conditions that would increase surgical risk, CRS is a reasonable approach. Surgery must be performed by an experienced surgical team; peritonectomy could be either limited to peritoneal surfaces visibly infiltrated by the disease or extended in the case of total parietal peritonectomy [48]. There is some controversy about the implementation of these two strategies. In a controlled study, the reported 5-year survival rates of selective versus parietal peritonectomy were $40 \%$ and $63.9 \%$, respectively $(\mathrm{P}=0.0269)$ [49]. The complete parietal peritonectomy was performed without increasing post-surgical morbidity or mortality.

MPM has a specific pattern of intraperitoneal dissemination. Parietal peritoneal surfaces are typically diffusely involved, which may necessitate extensive peritonectomy. The perihepatic regions may pose a substantial challenge, specifically the posterior aspect of the hepatoduodenal ligament. The extensive involvement of small- and large-bowel mesenteries is generally not amenable to cytoreduction.

The presence of lymph node metastases has been demonstrated to be a negative prognostic factor for survival [50]. All enlarged lymph nodes should be removed and assessed histologically. Lymph node groups that have been recommended for histopathologic evaluation include the deep epigastric lymph nodes, external, internal and common iliac lymph nodes, as well as lymph nodes at the origin of the gastroepiploic vessels [50,51].

\section{CRS and HIPEC}

CRS with HIPEC has been recommended as the standard of care for patients with operable disease, and has been evaluated in a series of studies [7,8,29,50-64]. Details are reported in Table 2. CRS is the basis for the implementation of this therapeutic modality, whereas HIPEC offers enhanced therapeutic activity via distribution of high-dose intraperitoneal chemotherapy to all peritoneal surfaces in parallel with hyperthermia [24].

The aim of CRS prior to intraperitoneal chemotherapy is the achievement of complete resection of the macroscopic tumor and lysis of preexistent intra-abdominal adhesions, with the prospect of optimal exposure to intraperitoneal drugs. In this sequence of procedures, postponement of bowel reconstruction following resections until after the chemotherapy perfusion is considered, to reduce the risk of tumor cell seeding at anastomotic sites. Intraperitoneal therapy seems to be beneficial only in those residual tumor nodules smaller than $3 \mathrm{~mm}$. There are six different peritonectomy procedures that can be performed either separately or combined: greater omentectomy-splenectomy, left upper quadrant peritonectomy, right upper quadrant peritonectomy, lesser omentectomy-cholecystectomy with stripping of the omental bursa, pelvic peritonectomy with sleeve resection of the sigmoid colon, and antrectomy [65].

Hyperthermia has a direct cytotoxic effect caused by impaired DNA repair, denaturation of proteins, induction of heat-shock proteins, and apoptosis as well as inhibition of angiogenesis. In addition, hyperthermia acts synergistically with certain cytotoxic drugs, such as cisplatin, ifosfamide, melphalan, and mitomycin $\mathrm{C}$, whereas it may also diminish the systemic toxicity of some drugs (e.g., doxorubicin and cyclophosphamide) by increasing their alkylation and/or excretion. Although enhancement of penetration depth should theoretically apply for all drugs, this has only been proven for cisplatin (Table 3) [65].

Peritoneal expansion is applied in most centers to optimize exposure of the intra-abdominal organs and the parietal peritoneum to the perfusate. This can be achieved by several 
Table 2 Summary of CRS plus HIPEC studies in the treatment of MPM during the last 15 years (2003-2018)

\begin{tabular}{|c|c|c|c|c|c|c|c|c|c|}
\hline \multirow[t]{2}{*}{ Author [ref.] } & \multirow[t]{2}{*}{ Publication year } & \multirow[t]{2}{*}{$\mathrm{N}$} & \multirow{2}{*}{$\begin{array}{c}\text { Median } \\
\text { OS (months) }\end{array}$} & \multicolumn{5}{|c|}{ Survival rate (years) } & \multirow{2}{*}{$\begin{array}{l}\text { Perioperative } \\
\text { mortality (\%) }\end{array}$} \\
\hline & & & & 1 & 2 & 3 & 5 & 10 & \\
\hline Deraco et al [52] & 2003 & 61 & & & & & 54 & & 0 \\
\hline Feldman et al [8] & 2003 & 49 & 92 & 86 & 77 & 59 & 59 & & 0 \\
\hline Yan et al [51] & 2006 & 100 & 52 & 78 & & 55 & 46 & & 5 \\
\hline Deraco et al [53] & 2006 & 49 & & 88 & 74 & 65 & 57 & & \\
\hline Baratti et al [29] & 2007 & 60 & & & & & 53.7 & & \\
\hline Yan et al [54] & 2007 & 70 & 59 & 82 & 67 & 57 & 49 & & 3 \\
\hline Hesdorffer et al [55] & 2008 & 27 & 70 & & & 67 & & & 0 \\
\hline Yan et al [7] & 2009 & 401 & 53 & 81 & & 60 & 47 & & 2.0 \\
\hline Baratti et al [50] & 2010 & 83 & 44 & & & & 49.5 & 45.5 & 2.4 \\
\hline Kluger et al [56] & & 47 & 54.9 & 80.9 & & 61.7 & 48.9 & & 2 \\
\hline Yan et al [57] & 2011 & 294 & 67 & 83 & 62 & 52 & & & \\
\hline Cao et al [58] & 2012 & 294 & 67 & 83 & 62 & 52 & & & 2 \\
\hline Alexander et al [59] & 2013 & 211 & 38.4 & & & & 41 & 26 & 2.3 \\
\hline Haslinger et al [60] & 2013 & 112 & 63.2 & & & & 91.3 & & 2.7 \\
\hline Baratti et al [61] & 2013 & 108 & 63.2 & & & & 52.4 & 44.6 & 1.9 \\
\hline Schaub et al [62] & 2013 & 104 & 52 & & & 58 & 46 & & \\
\hline Deraco et al [63] & 2013 & 116 & & & & & 49 & & 2.6 \\
\hline Magge et al [64] & 2014 & 65 & 46.2 & 77 & 57 & & 39 & & 6.0 \\
\hline
\end{tabular}

Table 3 Interaction between hyperthermia and cytotoxic drugs used during HIPEC

\begin{tabular}{ll}
\hline Agent & Synergism \\
\hline Mitomycin C & Yes (linear $\left.\geq 39^{\circ} \mathrm{C}\right)$ \\
Cisplatin & Yes $\left(\right.$ linear $\left.\geq 39^{\circ} \mathrm{C}\right)$ \\
Melphalan & Yes (linear $\left.\geq 39^{\circ} \mathrm{C}\right)$ \\
Mitoxantrone & Yes $\left(\right.$ linear $\left.\geq 39^{\circ} \mathrm{C}\right)$ \\
Bleomycin & Yes $\left(\right.$ threshold $\left.\geq 42^{\circ} \mathrm{C}\right)$ \\
Doxorubicin & Yes $\left(\right.$ threshold $\left.\geq 42^{\circ} \mathrm{C}\right)$ \\
\hline Taxanes & No \\
5-Fluorouracil & No \\
\hline
\end{tabular}

HIPEC, hyperthermic intraperitoneal chemotherapy

available methods. In the coliseum technique, the skin of the abdomen is attached to a retractor ring, above the laparotomy wound. The abdominal cavity is covered with a plastic sheet with a small opening in the centre allowing entrance for the surgeon's hand to stir the abdominal contents. A "peritoneal access device" to achieve optimal peritoneal expansion has also been described. Peritoneal expansion based on this technique permits large volumes of perfusion fluid to be added, enabling the small bowel to float in the cavity expander. Finally, there is a perfusion system in which the abdomen is closed during perfusion by a running suture of the skin. Thus, the whole peritoneal surface is exposed and drug spillage and heat loss are prevented [65].

Preoperative evaluation should determine whether optimal or complete CRS is feasible. Contraindications for CRS and HIPEC include severe cardiac, pulmonary, hepatic or renal dysfunction, and poor performance status [66]. In addition, high-volume peritoneal disease, extensive involvement of small bowel or mesentery, large tumor masses in the lesser omentum, disease burden outside the peritoneal cavity, and para-aortic lymph node metastases are contraindications, as they reduce the probability of optimal or complete CRS and are associated with poor outcomes [67].

The need to improve patient selection has led to various prognostic scoring systems that evaluate candidates preoperatively for CRS and HIPEC. The preoperative parameters used in those scoring systems include histopathology, symptoms, and tumor burden. Overall, the risks of perioperative morbidity and mortality, analogous to those of major gastrointestinal surgeries, should be weighed against the anticipated survival benefit, which depends on the extent of the disease and the feasibility of achieving complete CRS [67].

Immediately following a CRS and prior to intestinal reconstruction and abdominal closure, the abdomen and pelvis must be prepared for HIPEC, achieving complete hemostasis. Mechanical cleansing of the peritoneal space by irrigation should be performed, followed by HIPEC in patients who undergo 
complete or near-complete cytoreduction. No randomized clinical trials has been conducted for the comparison of the several available HIPEC chemotherapy regimens. Retrospective reports have described high-dose cisplatin $\left(250 \mathrm{mg} / \mathrm{m}^{2}\right)$ monotherapy $[8,59,62,68-70]$ or dual therapy, such as cisplatin combined with either doxorubicin [7,51,53,54,57,58,61,63,71-75] or mitomycin [50,52,55,56,76-78].

In a multi-institutional retrospective analysis of 405 patients with MPM treated with CRS-HIPEC [7], HIPEC, mainly in combination with cisplatin and doxorubicin, was administered to $92 \%$ of participants. An additional 23\% subsequently received systemic chemotherapy, mainly with paclitaxel, in an adjuvant setting during the early postoperative period (days 1-5). Median OS was 53 months and 5-year survival was achieved by $47 \%$. Likewise, a recent meta-analysis of 20 studies, which included 1047 patients with MPM treated with CRS-HIPEC, reported a 5-year survival of $42 \%$ in the $67 \%$ of patient who underwent complete or near-complete cytoreduction prior to HIPEC [79]. Myelosuppression is a common complication associated with HIPEC, while laparotomy and CRS may cause wound infections, prolonged ileus, bowel obstruction, urinary tract infections, sepsis, and fistula formation. Despite these known side effects of CRSHIPEC, the benefit is significant, with median OS and 5-year survival rates of 29.5-100 months and $17-91.3 \%$, respectively (Table 2). This broad range is obviously explained by the widely heterogeneous nature of differing study populations, tumor biology and experience of the surgical team. CRS and HIPEC were also effective in the management of variable origins of peritoneal carcinomas and malignant ascites [60,80-83], as well as in disease recurrence[84].

Early postoperative intraperitoneal chemotherapy with paclitaxel, combined with systemic chemotherapy, has been reported to be beneficial. The recommended intraperitoneal/ systemic chemotherapy regimens are similar to those implemented in ovarian cancer [85].

\section{Systemic chemotherapy}

CRS-HIPEC is considered as the standard first-line treatment for MPM, whereas systemic chemotherapy is the alternative approach for inoperable/unsuitable patients or for those patients who seek non-surgical management. Perioperative systemic chemotherapy has also been recommended in those with high-risk histology or extensive disease.

Limited clinical trials have been performed for the evaluation of systemic chemotherapy in patients with MPM[66]. Despite the fact that pleural and peritoneal mesothelioma are distinct, especially in terms of biology, the effectiveness of chemotherapeutic agents is similar for both entities. Neither single-agent systemic chemotherapy nor combinations are effective, and they correlate with a poor response rate of less than $15-20 \%$ [3]. The pemetrexed/cisplatin combination is approved for the treatment of MPM, based on the definitive phase III trial by Vogelzang et al [86].

The efficacy of pemetrexed for MPM, either alone or in combination with cisplatin, was reported in two studies[87,88]. The estimated median OS times were 8.7 and 13.1 months for pemetrexed monotherapy and the doublet systemic chemotherapy with cisplatin, respectively. There were no significant differences in the responses between chemotherapy-naïve patients and those previously treated with another agent. Pemetrexed was well tolerated, with low rates of reported grade 3 or 4 side effects, most often hematologic (2\%) or non-hematologic toxicities such as dehydration (7\%), nausea (5\%), and vomiting (5\%) [87]. A phase II trial revealed that the treatment with pemetrexed plus gemcitabine provides a significantly improved median OS of 26.8 months. However, the response rate of $15 \%$ still remains low [89]. Taking into consideration the increased incidence of severe toxicity and the inferior disease control rate with this combination, the option of pemetrexed with cisplatin/carboplatin is still considered as the standard first-line systemic treatment. The remaining drug combinations are reserved as a second-line option. Nevertheless, no therapies have shown a survival benefit as second-line treatments for relapsed or refractory disease. Failure to obtain disease control should prompt a switch to cisplatin combined with either irinotecan, or gemcitabine. Additional alternatives for second-line systemic treatment include the single agent vinorelbine, as well as the molecular agent tremelimumab, a monoclonal antibody that targets the cytotoxic T-lymphocyte antigen 4 (CTLA4). All these secondline therapies are still under investigation and enrollment in clinical trials should always be considered.

The use of perioperative systemic chemotherapy has also been investigated in MPM patients who underwent CRSHIPEC, while neoadjuvant chemotherapy was independently associated with a worse outcome [90]. The 5-year OS achieved with this approach was $40 \%$, compared to $56 \%, 67 \%$ and $62 \%$ in patients who had no systemic chemotherapy, or were treated in an adjuvant setting or perioperatively, respectively.

\section{Molecular therapy}

Deregulated expression of growth factors or proteins that function in downstream signaling pathways is crucial in the malignant transformation of mesothelial cells. A variety of molecular targets have been identified in MPM and relevant targeted agents have been investigated (Fig. 1). However, there is no consensus in the literature concerning the presence of epidermal growth factor receptor (EGFR) mutations in MPM. These mutations were identified in $31 \%$ of patients diagnosed with MPM [91], a similar rate to that reported for non-small cell lung cancer [92]. The presence of EGFR mutations is predictive of optimal cytoreduction - the only surrogate factor that predicts long-term survival [8] - treatment response and improved outcome as compared to the wild-type MPM [91]. On the other hand, in another study direct sequencing of the entire EGFR tyrosine kinase domain revealed that patients with MPM do not harbor somatic mutations in that domain that would make them sensitive to an EGFR tyrosine kinase inhibitor [93]. It is difficult to interpret the contradictory findings of these studies.

Overexpression of the closely interacting phosphoinositide 3-kinase (PI3K) and mammalian target of rapamycin (mTOR) 
pathways has emerged as an important molecular alteration that promotes a malignant phenotype of MPM [5]. Loss of phosphatase and tensin homolog (PTEN) function has been detected in $30-60 \%$ of cases [94]. Therefore, pharmacological inhibition of the PI3K-PTEN-AKT-mTOR pathways could be therapeutically beneficial in mesothelioma and clinical trials in this setting are ongoing [95]. In a case series of patients treated with CRS and HIPEC, upregulation in genes related to the PI3K and mTOR signaling pathways was associated with shorter OS [5]. Mesothelin is a cell surface glycoprotein expressed in both mesothelial and peritoneal cells. Three agents targeting mesothelin have been evaluated: SS1P, a recombinant immunotoxin targeting mesothelin; MORAb-009, a chimeric IgG1 anti-mesothelin monoclonal antibody; and CRS-207, a live-attenuated Listeria monocytogenes vector encoding human mesothelin [96]. Some novel mesothelin-targeted agents, including the immunocytokine interleukin (IL)-12, appear to have equivalent antitumor activity to SS1P in a murine model of MPM [97]. Furthermore, studies revealed that MORAb-009 blocks the binding of mesothelin to CA- 125 and thus could be a promising approach for the prevention of tumor metastasis [98]. Finally, the recombinant anti-mesothelin immunotoxin SS1(dsFv)PE38 demonstrated preclinical cytotoxic activity and is under clinical investigation [99].

An interesting molecular target is the glycoprotein MUC1. One study revealed that MUC1 was expressed in 90\% of patients with MPM and may carry some negative prognostic value [100]. Bromelain, a complex of proteolytic enzymes, has been proposed to target MUC1. Preliminary research indicates that chemoresistant peritoneal mesothelioma cell lines demonstrate increased sensitivity to bromelain combination therapy [101].

The NF2/Hippo signaling pathway appears to be disrupted in the majority of mesotheliomas [102]. Indeed, experimental animal models suggest that this event, together with a deficiency in CDKN2A, is crucial for the development of mesothelioma. Therefore, targeting molecules involved in the NF2/Hippo pathway is considered essential for the treatment of MPM. In addition, NF2 alterations lead to activation of focal adhesion kinase $(F A K)$ and merlin deficiency predicts sensitivity to FAK inhibitors [103].

The genomic profiling of MPM revealed recurrent mutations in the epigenetic regulatory genes BAP1, SETD2, and $D D X 3 X$ [104]. In this regard, transcriptional deregulation is a key oncogenic mechanism in mesothelial tumorigenesis that can lead to novel therapeutic implications for the treatment of MPM. Preclinical data suggest that inhibitors of epigenetic modifiers, including histone deacetylases and the histone methyltransferase EZH2, may be therapeutically beneficial in mesothelioma [105]. Those mesotheliomas that harbor inactivating events affecting both $B A P 1$ and other transcriptional regulators, including SETD2 and DDX3X, probably constitute a molecular subgroup with altered transcriptional programs that may respond therapeutically to these agents. Potentially targetable alterations have also been identified, such as MET, which can be targeted with the small molecule inhibitor cabozantinib, and WEE1 with AZD1775 for the subset of MPM harboring SETD2 inactivation [106]. Furthermore, in one study $A L K$ rearrangements were identified in 3\% of patients with MPM, mostly in younger women [107]. Interestingly, the $A L K$-rearranged cases lacked the typically genetic alterations present in MPM, i.e., BAP1, SETD2 or NF2. Therefore, oncogenic $A L K$ fusion may represent a distinct pathogenetic mechanism for a subset of patients with MPM who may be treated with ALK targeting agents.

\section{Immunotherapy}

Data from animal studies and small-cohort clinical trials suggested that malignant mesothelioma could be responsive to immunotherapy [108]. A chronic inflammatory reaction represented by infiltrating lymphocytes and plasma cells is associated with improved prognosis [109]. Indeed, tumor necrosis factor- $\alpha$, IL- 6 , interferon and granulocyte-macrophage colony-stimulating factor have been demonstrated to be effective in the treatment of mesothelioma [3]. In mice models, pulsed dendritic cells were shown to be powerful in controlling the growth of mesothelioma and may be implemented in the adjuvant setting to control local recurrence [110]. There is a genuine need for more phase II/III clinical trials to allow investigation in the field of these novel immunotherapies.

Cancer cells usually inhibit T-cell activation and evade immune surveillance. Activated T cells express CTLA-4. CTLA4-blocking monoclonal antibodies represent the standard of care of certain cancers, including melanoma [111]. In a phase II trial, the anti-CTLA-4 antibody tremelimumab has been evaluated as second-line treatment in mesothelioma [112]. In this study, a disease control rate of $31 \%$ and a PFS of 6 months were achieved, prompting further assessment of this agent in this setting.

Expression of programmed death-ligand 1 (PDL-1) allows cancers to escape from the host immune system by interaction with programmed cell death-1 (PD1), and has been demonstrated in mesothelioma tumor tissue [113]. This could lead to the development of a PD1/PDL-1 targeting agent for this disease. However, the predictive value of immunohistochemical screening regarding the response to anti-PD-L1 treatment for epithelioid MPM remains to be investigated.

\section{Prognostic factors}

MPM is a biologically heterogeneous tumor. Because of its rarity and the variability of treatment approaches, prognostic factors have not been clearly identified. Well-established prognostic factors are vital for optimizing the treatment of patients with MPM and multi-institutional registries must play a fundamental role in addressing this need [28,37]. Several patient- and treatment-related factors can be used for risk stratification in MPM patients undergoing CRS and HIPEC treatment $[59,64]$. One of the most consistent factors useful for predicting survival in MPM is the histologic type. It has been suggested that the epithelioid subtype is a favorable prognostic factor, while sarcomatoid and biphasic subtypes are associated 
with a dismal prognosis $[7,49,62,75]$. Accordingly, one study revealed that CRS-HIPEC in the sarcomatoid and biphasic groups may not be beneficial, with a median OS of 10.5 as compared to 51.5 months for those with a more favorable histology [64]. Survival is strongly affected by the completeness of cytoreduction [59,75]. In addition, outcomes from numerous studies identified stage as a prognostic factor [57]. A novel nomogram has been used for predicting survival, partly based on the peritoneal carcinomatosis index ranges[62]. In addition, it has been suggested that cell proliferation biomarkers such as Ki-67 may play a prognostic role in patients with MPM; high Ki-67 in association with a high peritoneal cancer index resulted in a median OS of only 10 months [57].

Older age is a negative predictive factor, even though the age of sample populations has varied among different studies. In general, patients older than 65 years have a dismal prognosis compared with those younger than 65 [64]. A possible explanation is that advanced age may increase post-surgical morbidity and mortality per se. There are certain differences in terms of the disease prognosis between the sexes, with female sex being associated with better survival in univariate analysis $[19,58,59]$. Less asbestos exposure, favorable histologic features, and the expression of estrogen receptors (ER- $\beta$ ) probably contribute to the better prognosis of female patients compared to male $[24,114]$.

A large, single-center experience revealed that preoperative thrombocytosis predicts shortened survival in patients with MPM who undergo CRS and HIPEC [115]. It seems that those with a high platelet count were more likely to undergo suboptimal debulking surgery. These data suggest that patients with baseline thrombocytosis are not good candidates for immediate CRS and HIPEC. Overall, thrombocytosis at diagnosis predicts an aggressive biological behavior of MPM, thus suggesting that platelets and other immunologic cytokines could represent potential targets for the development of new therapeutic agents.

\section{Concluding remarks}

MPM remains a rare disease with limited therapeutic options and a poor outcome. Immunohistochemical loss of nuclear BAP1 is highly specific in the distinction of MPM from benign mesothelial proliferations. In addition, $P A X 8$ immunostaining is valuable for differentiating MPM from serous ovarian cancer. The management of MPM requires meticulous patient selection and appropriate use of CRS and HIPEC in experienced centers. Optimal or suboptimal cytoreduction increases the chances of long-term survival in these patients. Systemic chemotherapy with the combination of pemetrexed and cisplatin in a first-line setting is reasonable for unresectable tumors, but the response remains limited. It is hoped that the molecular characterization of MPM tumors with novel sequencing technologies will lead to the identification of novel molecular targets in this disease. To date, overexpression of the PI3K/mTOR/AKT pathway appears to be a driver of the malignant phenotype of this tumor and represents an important target for the development of novel therapeutic interventions. Standardization of the treatment of peritoneal mesothelioma is crucial and will only be achieved through international collaboration and prospective clinical trials.

\section{References}

1. Yang H, Testa JR, Carbone M. Mesothelioma epidemiology, carcinogenesis, and pathogenesis. Curr Treat Options Oncol 2008;9:147-157.

2. Fasola G, Puglisi F, Follador A, Aita M, Di Terlizzi S, Belvedere O. Dramatic tumour response to pemetrexed single-agent in an elderly patient with malignant peritoneal mesothelioma: a case report. BMC Cancer 2006;6:289.

3. Robinson BW, Lake RA. Advances in malignant mesothelioma. $N$ Engl J Med 2005;353:1591-1603.

4. Liu S, Staats P, Lee M, Alexander HR, Burke AP. Diffuse mesothelioma of the peritoneum: correlation between histological and clinical parameters and survival in 73 patients. Pathology 2014;46:604-609.

5. Varghese S, Chen Z, Bartlett DL, et al. Activation of the phosphoinositide-3-kinase and mammalian target of rapamycin signaling pathways are associated with shortened survival in patients with malignant peritoneal mesothelioma. Cancer 2011;117:361-371.

6. Munkholm-Larsen S, Cao CQ, Yan TD. Malignant peritoneal mesothelioma. World J Gastrointest Surg 2009;1:38-48.

7. Yan TD, Deraco M, Baratti D, et al. Cytoreductive surgery and hyperthermic intraperitoneal chemotherapy for malignant peritoneal mesothelioma: multi-institutional experience. J Clin Oncol 2009;27:6237-6242.

8. Feldman AL, Libutti SK, Pingpank JF, et al. Analysis of factors associated with outcome in patients with malignant peritoneal mesothelioma undergoing surgical debulking and intraperitoneal chemotherapy. J Clin Oncol 2003;21:4560-4567.

9. Sugarbaker PH, Alderman R, Edwards G, et al. Prospective morbidity and mortality assessment of cytoreductive surgery plus perioperative intraperitoneal chemotherapy to treat peritoneal dissemination of appendiceal mucinous malignancy. Ann Surg Oncol 2006;13:635-644.

10. Kotova S, Wong RM, Cameron RB. New and emerging therapeutic options for malignant pleural mesothelioma: review of early clinical trials. Cancer Manag Res 2015;7:51-63.

11. Baratti D, Kusamura S, Deraco M. Diffuse malignant peritoneal mesothelioma: systematic review of clinical management and biological research. J Surg Oncol 2011;103:822-831.

12. Manzini VP, Recchia L, Cafferata M, et al. Malignant peritoneal mesothelioma: a multicenter study on 81 cases. Ann Oncol 2010;21:348-353.

13. Delgermaa V, Takahashi K, Park EK, Le GV, Hara T, Sorahan T. Global mesothelioma deaths reported to the World Health Organization between 1994 and 2008. Bull World Health Organ 2011;89:716-724, 724A-724C.

14. Blackham AU, Levine EA. Cytoreductive surgery with hyperthermic intraperitoneal chemotherapy for malignant peritoneal mesothelioma. European J Clin Med Oncol 2012;4:25-32.

15. Boffetta P. Epidemiology of peritoneal mesothelioma: a review. Ann Oncol 2007;18:985-990.

16. Kim J, Bhagwandin S, Labow DM. Malignant peritoneal mesothelioma: a review. Ann Transl Med 2017;5:236.

17. Marinaccio A, Corfiati M, Binazzi A, et al; ReNaM Working Group. 
The epidemiology of malignant mesothelioma in women: gender differences and modalities of asbestos exposure. Occup Environ Med 2018;75:254-262.

18. Rodríguez D, Cheung MC, Housri N, Koniaris LG. Malignant abdominal mesothelioma: defining the role of surgery. J Surg Oncol 2009;99:51-57.

19. Yan TD, Popa E, Brun EA, Cerruto CA, Sugarbaker PH. Sex difference in diffuse malignant peritoneal mesothelioma. Br J Surg 2006;93:1536-1542.

20. Carbone M, Ly BH, Dodson RF, et al. Malignant mesothelioma: facts, myths, and hypotheses. J Cell Physiol 2012;227:44-58.

21. Cao SB, Jin S, Cao JY, Shen J, Zhang JW, Yu Y. Colonic invasion induced by malignant peritoneal mesothelioma. Int J Colorectal Dis 2014;29:891-892.

22. Salemis NS, Tsiambas E, Gourgiotis S, Mela A, Karameris A, Tsohataridis E. Peritoneal mesothelioma presenting as an acute surgical abdomen due to jejunal perforation. J Dig Dis 2007;8:216-221.

23. Sugarbaker PH, Yan H, Grazi RV, Shmookler BM. Early localized peritoneal mesothelioma as an incidental finding at laparoscopy. Report of a case and implications regarding natural history of the disease. Cancer 2000;89:1279-1284.

24. Acherman YI, Welch LS, Bromley CM, Sugarbaker PH. Clinical presentation of peritoneal mesothelioma. Tumori 2003;89:269-273.

25. Haliloglu M, Hoffer FA, Fletcher BD. Malignant peritoneal mesothelioma in two pediatric patients: MR imaging findings. Pediatr Radiol 2000;30:251-255.

26. Low RN, Sebrechts CP, Barone RM, Muller W. Diffusion-weighted MRI of peritoneal tumors: comparison with conventional MRI and surgical and histopathologic findings-a feasibility study. AJR Am J Roentgenol 2009; 193:461-470.

27. Cao Q, Lu M, Heath J, et al. 18F-FDG PET/CT in a recurrent diffuse malignant peritoneal mesothelioma. Clin Nucl Med 2012;37:492-494.

28. Iversen LH, Rasmussen PC, Laurberg S. Value of laparoscopy before cytoreductive surgery and hyperthermic intraperitoneal chemotherapy for peritoneal carcinomatosis. $\mathrm{Br} \quad \mathrm{J}$ Surg 2013;100:285-292.

29. Baratti D, Kusamura S, Martinetti A, et al. Circulating CA125 in patients with peritoneal mesothelioma treated with cytoreductive surgery and intraperitoneal hyperthermic perfusion. Ann Surg Oncol 2007; 14:500-508.

30. Robinson BW, Creaney J, Lake R, et al. Mesothelin-family proteins and diagnosis of mesothelioma. Lancet 2003;362:1612-1616.

31. Levy AD, Arnáiz J, Shaw JC, Sobin LH. From the archives of the AFIP: primary peritoneal tumors: imaging features with pathologic correlation. Radiographics 2008;28:583-607.

32. Ordóñez NG. The diagnostic utility of immunohistochemistry and electron microscopy in distinguishing between peritoneal mesotheliomas and serous carcinomas: a comparative study. Mod Pathol 2006; 19:34-48.

33. Taube ET, Denkert C, Sehouli J, et al. Wilms tumor protein 1 (WT1)-- not only a diagnostic but also a prognostic marker in highgrade serous ovarian carcinoma. Gynecol Oncol 2016;140:494-502.

34. Ali RH, Kalloger SE, Santos JL, Swenerton KD, Gilks CB. Stage II to IV low-grade serous carcinoma of the ovary is associated with a poor prognosis: a clinicopathologic study of 32 patients from a population-based tumor registry. Int J Gynecol Pathol 2013;32:529-535.

35. Oe S, Hasegawa K, Nagase S, Kato R, Torii Y, Udagawa Y. Expression of podoplanin in epithelial ovarian carcinomas and its potential as a marker for clear cell adenocarcinoma. Int J Gynecol Pathol 2010;29:405-410.

36. Nofech-Mozes S, Khalifa MA, Ismiil N, et al. Immunophenotyping of serous carcinoma of the female genital tract. Mod Pathol 2008;21:1147-1155.
37. Taşkın S, Gümüş Y, Kiremitçi S, Kahraman K, Sertçelik A, Ortaç F. Malignant peritoneal mesothelioma presented as peritoneal adenocarcinoma or primary ovarian cancer: case series and review of the clinical and immunohistochemical features. Int J Clin Exp Pathol 2012;5:472-478.

38. Husain AN, Colby T, Ordonez N, et al; International Mesothelioma Interest Group. Guidelines for pathologic diagnosis of malignant mesothelioma: 2012 update of the consensus statement from the International Mesothelioma Interest Group. Arch Pathol Lab Med 2013;137:647-667.

39. Takeda M, Kasai T, Enomoto Y, et al. Comparison of genomic abnormality in malignant mesothelioma by the site of origin. J Clin Pathol 2014;67:1038-1043.

40. Bowen NJ, Logani S, Dickerson EB, et al. Emerging roles for PAX8 in ovarian cancer and endosalpingeal development. Gynecol Oncol 2007;104:331-337.

41. Ordóñez NG. Value of PAX8, PAX2, claudin-4, and $\mathrm{h}$-caldesmon immunostaining in distinguishing peritoneal epithelioid mesotheliomas from serous carcinomas. Mod Pathol 2013;26:553-562.

42. Andrici J, Jung J, Sheen A, et al. Loss of BAP1 expression is very rare in peritoneal and gynecologic serous adenocarcinomas and can be useful in the differential diagnosis with abdominal mesothelioma. Hum Pathol 2016;51:9-15.

43. Singhi AD, Krasinskas AM, Choudry HA, et al. The prognostic significance of BAP1, NF2, and CDKN2A in malignant peritoneal mesothelioma. Mod Pathol 2016;29:14-24.

44. Shinozaki-Ushiku A, Ushiku T, Morita S, Anraku M, Nakajima J, Fukayama M. Diagnostic utility of BAP1 and EZH2 expression in malignant mesothelioma. Histopathology 2017;70:722-733.

45. Chirac P, Maillet D, Leprêtre F, et al. Genomic copy number alterations in 33 malignant peritoneal mesothelioma analyzed by comparative genomic hybridization array. Hum Pathol 2016;55:72-82.

46. El Bezawy R, De Cesare M, Pennati M, et al. Antitumor activity of miR-34a in peritoneal mesothelioma relies on c-MET and AXL inhibition: persistent activation of ERK and AKT signaling as a possible cytoprotective mechanism. J Hematol Oncol 2017;10:19.

47. Cimino-Reale G, Gandellini P, Santambrogio F, Recagni M, Zaffaroni N, Folini M. miR-380-5p-mediated repression of TEP1 and TSPYL5 interferes with telomerase activity and favours the emergence of an "ALT-like" phenotype in diffuse malignant peritoneal mesothelioma cells. J Hematol Oncol 2017;10:140.

48. Tabrizian P, Jayakrishnan TT, Zacharias A, et al. Incorporation of diagnostic laparoscopy in the management algorithm for patients with peritoneal metastases: A multi-institutional analysis. J Surg Oncol 2015;111:1035-1040.

49. Baratti D, Kusamura S, Cabras AD, Deraco M. Cytoreductive surgery with selective versus complete parietal peritonectomy followed by hyperthermic intraperitoneal chemotherapy in patients with diffuse malignant peritoneal mesothelioma: a controlled study. Ann Surg Oncol 2012;19:1416-1424.

50. Baratti D, Kusamura S, Cabras AD, Laterza B, Balestra MR, Deraco M. Lymph node metastases in diffuse malignant peritoneal mesothelioma. Ann Surg Oncol 2010;17:45-53.

51. Yan TD, Yoo D, Sugarbaker PH. Significance of lymph node metastasis in patients with diffuse malignant peritoneal mesothelioma. Eur J Surg Oncol 2006;32:948-953.

52. Deraco M, De Simone M, Rossi CR, et al. An Italian Multicentric Phase II study on peritonectomy and intra peritoneal hyperthermic perfusion (IPHP) to treat patients with peritoneal mesothelioma. J Exp Clin Cancer Res 2003;22:41-45.

53. Deraco M, Nonaka D, Baratti D, et al. Prognostic analysis of clinicopathologic factors in 49 patients with diffuse malignant peritoneal mesothelioma treated with cytoreductive surgery and intraperitoneal hyperthermic perfusion. Ann Surg Oncol 
2006;13:229-237.

54. Yan TD, Edwards G, Alderman R, Marquardt CE, Sugarbaker PH. Morbidity and mortality assessment of cytoreductive surgery and perioperative intraperitoneal chemotherapy for diffuse malignant peritoneal mesothelioma-a prospective study of 70 consecutive cases. Ann Surg Oncol 2007;14:515-525.

55. Hesdorffer ME, Chabot JA, Keohan ML, et al. Combined resection, intraperitoneal chemotherapy, and whole abdominal radiation for the treatment of malignant peritoneal mesothelioma. Am J Clin Oncol 2008;31:49-54.

56. Kluger MD, Taub RN, Hesdorffer M, Jin Z, Chabot JA. Twostage operative cytoreduction and intraperitoneal chemotherapy for diffuse malignant peritoneal mesothelioma: Operative morbidity and mortality in phase I and II trials. Eur J Surg Oncol 2010;36:997-1003.

57. Yan TD, Deraco M, Elias D, et al; Peritoneal Surface Oncology Group. A novel tumor-node-metastasis (TNM) staging system of diffuse malignant peritoneal mesothelioma using outcome analysis of a multi-institutional database* . Cancer 2011;117:1855-1863.

58. Cao C, Yan TD, Deraco M, et al; Peritoneal Surface Malignancy Group. Importance of gender in diffuse malignant peritoneal mesothelioma. Ann Oncol 2012;23:1494-1498.

59. Alexander HR Jr, Bartlett DL, Pingpank JF, et al. Treatment factors associated with long-term survival after cytoreductive surgery and regional chemotherapy for patients with malignant peritoneal mesothelioma. Surgery 2013;153:779-786.

60. Haslinger M, Francescutti V, Attwood K, et al. A contemporary analysis of morbidity and outcomes in cytoreduction/hyperthermic intraperitoneal chemoperfusion. Cancer Med 2013;2:334-342.

61. Baratti D, Kusamura S, Cabras AD, Bertulli R, Hutanu I, Deraco M. Diffuse malignant peritoneal mesothelioma: longterm survival with complete cytoreductive surgery followed by hyperthermic intraperitoneal chemotherapy (HIPEC). Eur J Cancer 2013;49:3140-3148.

62. Schaub NP, Alimchandani M, Quezado M, et al. A novel nomogram for peritoneal mesothelioma predicts survival. Ann Surg Oncol 2013;20:555-561.

63. Deraco M, Baratti D, Hutanu I, Bertuli R, Kusamura S. The role of perioperative systemic chemotherapy in diffuse malignant peritoneal mesothelioma patients treated with cytoreductive surgery and hyperthermic intraperitoneal chemotherapy. Ann Surg Oncol 2013;20:1093-1100.

64. Magge D, Zenati MS, Austin F, et al. Malignant peritoneal mesothelioma: prognostic factors and oncologic outcome analysis. Ann Surg Oncol 2014;21:1159-1165.

65. Witkamp AJ, de Bree E, Van Goethem R, Zoetmulder FA. Rationale and techniques of intra-operative hyperthermic intraperitoneal chemotherapy. Cancer Treat Rev 2001;27:365-374.

66. Turner K, Varghese S, Alexander HR Jr. Current concepts in the evaluation and treatment of patients with diffuse malignant peritoneal mesothelioma. J Natl Compr Canc Netw 2012;10:49-57.

67. Spiliotis J, Halkia E, de Bree E. Treatment of peritoneal surface malignancies with hyperthermic intraperitoneal chemotherapycurrent perspectives. Curr Oncol 2016;23:e266-e275.

68. Park BJ, Alexander HR, Libutti SK, et al. Treatment of primary peritoneal mesothelioma by continuous hyperthermic peritoneal perfusion (CHPP). Ann Surg Oncol 1999;6:582-590.

69. Blackham AU, Shen P, Stewart JH, Russell GB, Levine EA. Cytoreductive surgery with intraperitoneal hyperthermic chemotherapy for malignant peritoneal mesothelioma: mitomycin versus cisplatin. Ann Surg Oncol 2010;17:2720-2727.

70. Wong J, Koch AL, Deneve JL, Fulp W, Tanvetyanon T, Dessureault S. Repeat cytoreductive surgery and heated intraperitoneal chemotherapy may offer survival benefit for intraperitoneal mesothelioma: a single institution experience. Ann Surg Oncol 2014;21:1480-1486.
71. Costamagna D, Scuderi S, Vaira M, Barone R, De Simone M. [Treatment of peritoneal mesothelioma using cytoreduction and intraperitoneal hyperthermic chemotherapy]. Tumori 2003;89:40-42.

72. Baratti D, Kusamura S, Nonaka D, Oliva GD, Laterza B, Deraco M. Multicystic and well-differentiated papillary peritoneal mesothelioma treated by surgical cytoreduction and hyperthermic intra-peritoneal chemotherapy (HIPEC). Ann Surg Oncol 2007; 14:2790-2797.

73. Yan TD, Brun EA, Cerruto CA, Haveric N, Chang D, Sugarbaker PH. Prognostic indicators for patients undergoing cytoreductive surgery and perioperative intraperitoneal chemotherapy for diffuse malignant peritoneal mesothelioma. Ann Surg Oncol 2007;14:41-49.

74. Gómez Portilla A, Cendoya I, Muriel J, et al. Malignant peritoneal mesothelioma. Our experienced with triple combined therapy: cytoreduction, intraperitoneal perioperative chemotherapy and hyperthermia. Cir Esp 2007;81:82-86.

75. Chua TC, Yan TD, Morris DL. Outcomes of cytoreductive surgery and hyperthermic intraperitoneal chemotherapy for peritoneal mesothelioma: the Australian experience. J Surg Oncol 2009;99:109-113.

76. Deraco M, Casali P, Inglese MG, et al. Peritoneal mesothelioma treated by induction chemotherapy, cytoreductive surgery, and intraperitoneal hyperthermic perfusion. I Surg Oncol 2003;83:147-153.

77. Brigand C, Monneuse O, Mohamed F, et al. Peritoneal mesothelioma treated by cytoreductive surgery and intraperitoneal hyperthermic chemotherapy: results of a prospective study. Ann Surg Oncol 2006;13:405-412.

78. Passot G, Cotte E, Brigand C, et al. Peritoneal mesothelioma: treatment with cytoreductive surgery combined with hyperthermic intraperitoneal chemotherapy. J Chir (Paris) 2008;145:447-453.

79. Helm JH, Miura JT, Glenn JA, et al. Cytoreductive surgery and hyperthermic intraperitoneal chemotherapy for malignant peritoneal mesothelioma: a systematic review and meta-analysis. Ann Surg Oncol 2015;22:1686-1693.

80. Levine EA, Stewart JH 4th, Shen P, Russell GB, Loggie BL, Votanopoulos KI. Intraperitoneal chemotherapy for peritoneal surface malignancy: experience with 1,000 patients. J Am Coll Surg 2014;218:573-585.

81. Randle RW, Swett KR, Swords DS, et al. Efficacy of cytoreductive surgery with hyperthermic intraperitoneal chemotherapy in the management of malignant ascites. Ann Surg Oncol 2014;21:1474-1479.

82. Votanopoulos KI, Ihemelandu C, Shen P, Stewart JH, Russell GB, Levine EA. Outcomes of repeat cytoreductive surgery with hyperthermic intraperitoneal chemotherapy for the treatment of peritoneal surface malignancy. J Am Coll Surg 2012;215:412-417.

83. Chua TC, Quinn LE, Zhao J, Morris DL. Iterative cytoreductive surgery and hyperthermic intraperitoneal chemotherapy for recurrent peritoneal metastases. J Surg Oncol 2013;108:81-88.

84. Ihemelandu C, Bijelic L, Sugarbaker PH. Iterative cytoreductive surgery and hyperthermic intraperitoneal chemotherapy for recurrent or progressive diffuse malignant peritoneal mesothelioma: clinicopathologic characteristics and survival outcome. Ann Surg Oncol 2015;22:1680-1685.

85. Landrum LM, Java J, Mathews CA, et al. Prognostic factors for stage III epithelial ovarian cancer treated with intraperitoneal chemotherapy: a Gynecologic Oncology Group study. Gynecol Oncol 2013;130:12-18.

86. Vogelzang NJ, Rusthoven JJ, Symanowski J, et al. Phase III study of pemetrexed in combination with cisplatin versus cisplatin alone in patients with malignant pleural mesothelioma. J Clin Oncol 2003;21:2636-2644. 
87. Carteni G, Manegold C, Garcia GM, et al. Malignant peritoneal mesothelioma-Results from the International Expanded Access Program using pemetrexed alone or in combination with a platinum agent. Lung Cancer 2009;64:211-218.

88. Jänne PA, Wozniak AJ, Belani CP, et al. Open-label study of pemetrexed alone or in combination with cisplatin for the treatment of patients with peritoneal mesothelioma: outcomes of an expanded access program. Clin Lung Cancer 2005;7:40-46.

89. Simon GR, Verschraegen CF, Jänne PA, et al. Pemetrexed plus gemcitabine as first-line chemotherapy for patients with peritoneal mesothelioma: final report of a phase II trial. J Clin Oncol 2008;26:3567-3572.

90. Kepenekian V, Elias D, Passot G, et al; French Network for Rare Peritoneal Malignancies (RENAPE). Diffuse malignant peritoneal mesothelioma: Evaluation of systemic chemotherapy with comprehensive treatment through the RENAPE Database: Multi-Institutional Retrospective Study. Eur J Cancer 2016;65:6979.

91. Foster JM, Radhakrishna U, Govindarajan V, et al. Clinical implications of novel activating EGFR mutations in malignant peritoneal mesothelioma. World J Surg Oncol 2010;8:88.

92. Foster JM, Gatalica Z, Lilleberg S, Haynatzki G, Loggie BW. Novel and existing mutations in the tyrosine kinase domain of the epidermal growth factor receptor are predictors of optimal resectability in malignant peritoneal mesothelioma. Ann Surg Oncol 2009;16:152-158.

93. Kalra N, Ashai A, Xi L, et al. Patients with peritoneal mesothelioma lack epidermal growth factor receptor tyrosine kinase mutations that would make them sensitive to tyrosine kinase inhibitors. Oncol Rep 2012;27:1794-1800.

94. Agarwal V, Campbell A, Beaumont KL, Cawkwell L, Lind MJ. PTEN protein expression in malignant pleural mesothelioma. Tumour Biol 2013;34:847-851.

95. Dolly SO, Migali C, Tunariu N, et al. Indolent peritoneal mesothelioma: PI3K-mTOR inhibitors as a novel therapeutic strategy. ESMO Open 2017;2:e000101.

96. Hassan R, Ho M. Mesothelin targeted cancer immunotherapy. Eur J Cancer 2008;44:46-53.

97. Kim H, Gao W, Ho M. Novel immunocytokine IL12-SS1 (Fv) inhibits mesothelioma tumor growth in nude mice. PLoS One 2013;8:e81919.

98. Ma J, Tang WK, Esser L, Pastan I, Xia D. Recognition of mesothelin by the therapeutic antibody MORAb-009: structural and mechanistic insights. J Biol Chem 2012;287:33123-33131.

99. Li Q, Verschraegen CF, Mendoza J, Hassan R. Cytotoxic activity of the recombinant anti-mesothelin immunotoxin, SS1(dsFv)PE38, towards tumor cell lines established from ascites of patients with peritoneal mesotheliomas. Anticancer Res 2004;24:1327-1335.

100. Pillai K, Pourgholami MH, Chua TC, Morris DL. MUC1 has prognostic significance in malignant peritoneal mesothelioma. Int J Biol Markers 2013;28:303-312.

101. Pillai K, Akhter J, Chua TC, Morris DL. Anticancer property of bromelain with therapeutic potential in malignant peritoneal mesothelioma. Cancer Invest 2013;31:241-250.
102. Murakami H, Mizuno T, Taniguchi T, et al. LATS2 is a tumor suppressor gene of malignant mesothelioma. Cancer Res 2011;71:873-883.

103. Shapiro IM, Kolev VN, Vidal CM, et al. Merlin deficiency predicts FAK inhibitor sensitivity: a synthetic lethal relationship. Sci Transl Med 2014;6:237ra68.

104. Joseph NM, Chen YY, Nasr A, et al. Genomic profiling of malignant peritoneal mesothelioma reveals recurrent alterations in epigenetic regulatory genes BAP1, SETD2, and DDX3X. Mod Pathol 2017;30:246-254.

105. LaFave LM, Béguelin W, Koche R, et al. Loss of BAP1 function leads to EZH2-dependent transformation. Nat Med 2015;21:1344-1349.

106. Pfister SX, Markkanen E, Jiang Y, et al. Inhibiting WEE1 selectively kills histone H3K36me3-deficient cancers by dNTP starvation. Cancer Cell 2015;28:557-568.

107. Hung YP, Dong F, Watkins JC, et al. Identification of ALK Rearrangements in Malignant Peritoneal Mesothelioma. JAMA Oncol 2018;4:235-238.

108. Cao S, Jin S, Cao J, et al. Advances in malignant peritoneal mesothelioma. Int J Colorectal Dis 2015;30:1-10.

109. Suzuki K, Kadota K, Sima CS, et al. Chronic inflammation in tumor stroma is an independent predictor of prolonged survival in epithelioid malignant pleural mesothelioma patients. Cancer Immunol Immunother 2011;60:1721-1728.

110. Hegmans JP, Hemmes A, Aerts JG, Hoogsteden HC, Lambrecht BN. Immunotherapy of murine malignant mesothelioma using tumor lysate-pulsed dendritic cells. Am J Respir Crit Care Med 2005;171:1168-1177.

111. Callahan MK, Wolchok JD. At the bedside: CTLA-4- and PD1-blocking antibodies in cancer immunotherapy. J Leukoc Biol 2013;94:41-53.

112. Calabrò L, Morra A, Fonsatti E, et al. Tremelimumab for patients with chemotherapy-resistant advanced malignant mesothelioma: an open-label, single-arm, phase 2 trial. Lancet Oncol 2013;14:1104-1111.

113. Mansfield AS, Roden AC, Peikert T, et al. B7-H1 expression in malignant pleural mesothelioma is associated with sarcomatoid histology and poor prognosis. J Thorac Oncol 2014;9:1036-1040.

114. Pillai K, Pourgholami MH, Chua TC, Morris DL. Oestrogen receptors are prognostic factors in malignant peritoneal mesothelioma. J Cancer Res Clin Oncol 2013;139:987-994.

115. Li YC, Khashab T, Terhune J, et al. Preoperative thrombocytosis predicts shortened survival in patients with malignant peritoneal mesothelioma undergoing operative cytoreduction and hyperthermic intraperitoneal chemotherapy. Ann Surg Oncol 2017; 24:2259-2265.

116. Kato S, Tomson BN, Buys TP, Elkin SK, Carter JL, Kurzrock R. Genomic landscape of malignant mesotheliomas. Mol Cancer Ther 2016;15:2498-2507.

117. Tandon RT, Jimenez-Cortez Y, Taub R, Borczuk AC. Immunohistochemistry in peritoneal mesothelioma: a single-center experience of 244 cases. Arch Pathol Lab Med 2018;142:236242. 